Pacific

Journal of

Mathematics

THE JOHNSON-MORITA THEORY FOR THE RING OF FRICKE CHARACTERS OF FREE GROUPS

TAKAO SATOH 


\title{
THE JOHNSON-MORITA THEORY FOR THE RING OF FRICKE CHARACTERS OF FREE GROUPS
}

\author{
TAKAO SATOH
}

For a free group $F_{n}$ of rank $n$, we consider the ring $\mathfrak{X}_{\mathbb{Q}}\left(F_{n}\right)$ of $\operatorname{SL}(2, \mathbb{C})$ characters of $F_{n}$ generated by all Fricke characters $\operatorname{tr} x$ for $x \in F_{n}$. Its ideal $J$ generated by $\operatorname{tr} x-2$ for all $x \in F_{n}$ is Aut $F_{n}$-invariant. We denote by $\mathcal{E}_{n}(1)$ the subgroup of the automorphism group Aut $F_{n}$ of $F_{n}$ consisting of all automorphisms which act on $J / J^{2}$ trivially. The group $\mathcal{E}_{n}(1)$ is regarded as a Fricke character analogue of the IA-automorphism group of $F_{n}$ and the Torelli subgroup of the mapping class group of a surface. In our previous work, we constructed a homomorphism $\eta_{1}$ from $\mathcal{E}_{n}(1)$ into $\operatorname{Hom}_{\mathbb{Q}}\left(J / J^{2}, J^{2} / J^{3}\right)$ as a Fricke character analogue of the first Johnson homomorphisms of the mapping class group and Aut $\boldsymbol{F}_{\boldsymbol{n}}$.

In this paper, according to Morita's work for the extension of the first Johnson homomorphism of the mapping class group, we extend $\eta_{1}$ to Aut $F_{n}$ as a crossed homomorphism. We see that the obtained crossed homomorphism $\eta$ is not null cohomologous. We also compute the images of Nielsen's generators of Aut $F_{n}$ by $\eta$.

1. Introduction 443

2. Preliminaries 447

3. An extension of $\eta_{1}$ as a crossed homomorphism 452

4. Nontriviality of $\eta$ as a 1-cocycle 457

5. The image of the crossed homomorphism $\eta \quad 458$

Acknowledgments $\quad 460$

References $\quad 460$

\section{Introduction}

In a series of works, Dennis Johnson [1980; 1983; 1985a; 1985b] established a remarkable method to investigate the group structure of the Torelli subgroup of the mapping class group of a surface. In particular, he constructed in [Johnson 1985b] a homomorphism $\tau$ to determine the abelianization of the Torelli subgroup.

MSC2010: primary 20F28; secondary 20J06.

Keywords: ring of Fricke characters, automorphism group of free groups, IA-automorphism group, Andreadakis-Johnson filtration, Johnson homomorphisms. 
Today, his homomorphism $\tau$ is called the first Johnson homomorphism and has been generalized to those of higher degrees. Over the last two decades, good progress was made in the study of the Johnson homomorphisms of mapping class groups through the work of many authors including Morita [1993a], Hain [1997] and others.

Let $F_{n}$ be a free group generated by $x_{1}, x_{2}, \ldots, x_{n}$. As is well known, for any $g \geq 1$, the mapping class group $\mathcal{M}_{g, 1}$ of a compact oriented surface $\Sigma_{g, 1}$ with one boundary component can be embedded into Aut $F_{2 g}$ by a classical work of Dehn and Nielsen. This embedding is induced from the action of $\mathcal{M}_{g, 1}$ on the fundamental group of $\Sigma_{g, 1}$. The definition of the Johnson homomorphisms of $\mathcal{M}_{g, 1}$ can be naturally generalized to those of Aut $F_{n}$. Let $H$ be the abelianization of $F_{n}$. The kernel of the homomorphism Aut $F_{n} \rightarrow$ Aut $H \cong \mathrm{GL}(n, \mathbb{Z})$ induced from the action of Aut $F_{n}$ on $H$, is called the IA-automorphism group of $F_{n}$ and is denoted by $\mathrm{IA}_{n}$. The group $\mathrm{IA}_{n}$ is a free group analogue of the Torelli subgroup $\mathcal{I}_{g, 1}$ of $\mathcal{M}_{g, 1}$. Andreadakis [1965] introduced a central filtration

$$
\mathrm{IA}_{n}=\mathcal{A}_{n}(1) \supset \mathcal{A}_{n}(2) \supset \cdots
$$

of $\mathrm{IA}_{n}$, and showed that each graded quotient $\operatorname{gr}^{k} \mathcal{A}_{n}:=\mathcal{A}_{n}(k) / \mathcal{A}_{n}(k+1)$ is a free abelian group of finite rank. We call the above filtration the Andreadakis-Johnson filtration of Aut $F_{n}$. Johnson studied this type of filtration for the mapping class groups in 1980s. The general linear group $\operatorname{GL}(n, \mathbb{Z})$ naturally acts on each $\mathrm{gr}^{k} \mathcal{A}_{n}$. In order to investigate the $\mathrm{GL}(n, \mathbb{Z})$-module structure of $\mathrm{gr}^{k} \mathcal{A}_{n}$, the $k$-th Johnson homomorphism

$$
\tau_{k}: \mathrm{gr}^{k} \mathcal{A}_{n} \rightarrow H^{*} \otimes_{\mathbb{Z}} \mathcal{L}_{n}(k+1)
$$

is a powerful and useful tool. However, even the GL $(n, \mathbb{Q})$-structure of $\left(\mathrm{gr}^{k} \mathcal{A}_{n}\right) \otimes_{\mathbb{Z}}$ $\mathbb{Q}$ is not determined in general (see Section 2C for notation, and [Satoh 2009; 2013b] for basic materials concerning the Andreadakis-Johnson filtration and the Johnson homomorphisms of Aut $F_{n}$ ).

Now, we study a Fricke character analogue of the Andreadakis-Johnson filtration and the Johnson homomorphisms of Aut $F_{n}$. Let $R\left(F_{n}\right)$ be the set of all SL(2, C)representations of $F_{n}$, and $\mathcal{F}\left(R\left(F_{n}\right), \mathbb{C}\right)$ the set of all complex-valued functions on $R\left(F_{n}\right)$. Then $\mathcal{F}\left(R\left(F_{n}\right), \mathbb{C}\right)$ naturally has the $\mathbb{C}$-algebra structure coming from the pointwise product, and Aut $F_{n}$ naturally acts on $\mathcal{F}\left(R\left(F_{n}\right), \mathbb{C}\right)$ from the right. For any $x \in F_{n}$, define $\operatorname{tr} x \in \mathcal{F}\left(R\left(F_{n}\right), \mathbb{C}\right)$ by

$$
(\operatorname{tr} x)(\rho):=\operatorname{tr} \rho(x), \quad \rho \in R\left(F_{n}\right) .
$$

Here $\operatorname{tr}$ on the right-hand side means the usual trace of $2 \times 2$ matrix $\rho(x)$. The element tr $x$ is called the Fricke character of $x \in F_{n}$. Classically, Fricke characters were introduced by Fricke and Klein [1897] to study the moduli space of compact 
Riemann surfaces. In this paper, however, we focus on purely algebraic properties of the Fricke characters. Let $\mathfrak{X}_{\mathbb{Q}}\left(F_{n}\right)$ be the $\mathbb{Q}$-subalgebra of $\mathcal{F}\left(R\left(F_{n}\right), \mathbb{C}\right)$ generated by all $\operatorname{tr} x$ for $x \in F_{n}$. We call $\mathfrak{X}_{\mathbb{Q}}\left(F_{n}\right)$ the ring of Fricke characters of $F_{n}$ over $\mathbb{Q}$. Horowitz [1972] showed that $\mathfrak{X}_{\mathbb{Q}}\left(F_{n}\right)$ is finitely generated by

$$
\left\{\operatorname{tr} x_{i_{1}} \cdots x_{i_{l}} \mid 1 \leq l \leq 3,1 \leq i_{1}<i_{2}<\cdots<i_{l} \leq n\right\} .
$$

In order to establish the Johnson-Morita theory for $\mathfrak{X}_{\mathbb{Q}}\left(F_{n}\right)$, to begin with, we need a descending filtration of $\mathfrak{X}_{\mathbb{Q}}\left(F_{n}\right)$ consisting of Aut $F_{n}$-invariant ideals. Consider an ideal

$$
J:=\left(\operatorname{tr}^{\prime} x_{i_{1}} \cdots x_{i_{l}} \mid 1 \leq l \leq 3,1 \leq i_{1}<i_{2}<\cdots<i_{l} \leq n\right) \subset \mathfrak{X}_{\mathbb{Q}}\left(F_{n}\right),
$$

where $\operatorname{tr}^{\prime} x:=\operatorname{tr} x-2$ for any $x \in F_{n}$. Magnus [1980] showed that $J$ is Aut $F_{n}$ invariant for the case where $n=3$, and studied a representation of the quotient group of $\mathrm{IA}_{3}$ by the inner automorphism group Inn $F_{3}$ of $F_{3}$. On the other hand, it is easily seen that $J$ is Aut $F_{n}$-invariant for general $n \geq 3$. In this paper we use this ideal and the descending filtration

$$
J \supset J^{2} \supset J^{3} \supset \cdots
$$

in order to define a descending filtration of Aut $F_{n}$ as an analogy of the AndreadakisJohnson filtration. Although each graded quotient $\operatorname{gr}^{k} J:=J^{k} / J^{k+1}$ is an Aut $F_{n^{-}}$ invariant finite-dimensional $\mathbb{Q}$-vector space, by combinatorial complexities, it is quite difficult to give a basis of $\mathrm{gr}^{k} J$ for $n \geq 3$ in general. In [Hatakenaka and Satoh 2014] — henceforth abbreviated [HS 2014] — we explicitly give bases of $\mathrm{gr}^{k} J$ for $k=1$ and 2 (see Section 4 for details).

For any $k \geq 1$, set

$$
\mathcal{E}_{n}(k):=\operatorname{ker}\left(\operatorname{Aut} F_{n} \rightarrow \operatorname{Aut}\left(J / J^{k+1}\right)\right),
$$

where the homomorphism is induced by the action of Aut $F_{n}$ on $J / J^{k+1}$. The groups $\mathcal{E}_{n}(k)$ define a descending filtration

$$
\mathcal{E}_{n}(1) \supset \mathcal{E}_{n}(2) \supset \cdots
$$

of Aut $F_{n}$. In [HS 2014], we showed this filtration is central, and $\mathcal{A}_{n}(2 k) \subset \mathcal{E}_{n}(k)$ for any $k \geq 1$. Furthermore, we determined the first term $\mathcal{E}_{n}(1)$ to be $\operatorname{Inn} F_{n} \cdot \mathcal{A}_{n}(2)$ where $\operatorname{Inn} F_{n}$ is the inner automorphism group of $F_{n}$. Thus, each graded quotient $\operatorname{gr}^{k} \mathcal{E}_{n}:=\mathcal{E}_{n}(k) / \mathcal{E}_{n}(k+1)$ is an abelian group for any $k \geq 1$. In order to study the structures of $\mathrm{gr}^{k} \mathcal{E}_{n}$, we have introduced homomorphisms

$$
\eta_{k}: \mathrm{gr}^{k} \mathcal{E}_{n} \rightarrow \operatorname{Hom}_{\mathbb{Q}}\left(\mathrm{gr}^{1} J, \mathrm{gr}^{k+1} J\right)
$$

defined by

$$
\sigma\left(\bmod \mathcal{E}_{n}(k+1)\right) \mapsto\left(f\left(\bmod J^{2}\right) \mapsto f^{\sigma}-f\left(\bmod J^{k+1}\right)\right) .
$$


The homomorphism $\eta_{k}$ is a Fricke character analogue of the $k$-th Johnson homomorphism $\tau_{k}$. In [HS 2014], we showed that each $\eta_{k}$ is an Aut $F_{n} / \mathcal{E}_{n}(1)$-equivariant injective homomorphism. This implies that each of $\mathrm{gr}^{k} \mathcal{E}_{n}$ is torsion-free, and that $\operatorname{dim}_{\mathbb{Q}}\left(\operatorname{gr}^{k} \mathcal{E}_{n} \otimes_{\mathbb{Z}} \mathbb{Q}\right)<\infty$.

In this paper, we concentrate on the homomorphism

$$
\tilde{\eta}_{1}: \mathcal{E}_{n}(1) \rightarrow \mathrm{gr}^{1} \mathcal{E}_{n} \stackrel{\eta_{1}}{\rightarrow} \operatorname{Hom}_{\mathbb{Q}}\left(\mathrm{gr}^{1} J, \mathrm{gr}^{2} J\right) .
$$

Morita [1993b] showed that the composition

$$
\mathcal{I}_{g, 1} \stackrel{\tau_{1}}{\rightarrow} H^{*} \otimes_{\mathbb{Z}} \mathcal{L}_{2 g}(k+1) \rightarrow\left(H^{*} \otimes_{\mathbb{Z}} \mathcal{L}_{2 g}(k+1)\right) \otimes_{\mathbb{Z}} \mathbb{Z}\left[\frac{1}{2}\right]
$$

of the first Johnson homomorphism of the mapping class group $\mathcal{M}_{g, 1}$ the natural projection naturally extends to $\mathcal{M}_{g, 1}$ as a crossed homomorphism. He also showed that this extension is unique up to 1 -coboundary. Here $\mathbb{Z}\left[\frac{1}{2}\right]$ means a subring of $\mathbb{Q}$ obtained from $\mathbb{Z}$ by attaching $\frac{1}{2}$. The analogous result for Aut $F_{n}$ was obtained by Kawazumi [2006], who showed the composition

$$
\mathrm{IA}_{n} \rightarrow \mathrm{gr}^{1} \mathcal{A}_{n} \stackrel{\tau_{1}}{\rightarrow} H^{*} \otimes_{\mathbb{Z}} \mathcal{L}_{n}(k+1) \rightarrow\left(H^{*} \otimes_{\mathbb{Z}} \mathcal{L}_{n}(k+1)\right) \otimes_{\mathbb{Z}} \mathbb{Z}\left[\frac{1}{2}\right]
$$

of the first Johnson homomorphism of Aut $F_{n}$ with the natural projection naturally and uniquely extends to Aut $F_{n}$ as a crossed homomorphism up to 1-coboundary. Furthermore, very recently, Day [2013] showed that each Johnson homomorphism $\tau_{k}$ of Aut $F_{n}$ can be extended to Aut $F_{n}$ as a crossed homomorphism.

The main purpose of the paper is to give a Fricke character analogue of these results. Namely, according to Morita's work, we extend $\tilde{\eta}_{1}$ to Aut $F_{n}$ as a crossed homomorphism:

Theorem 3.6. There is a crossed homomorphism $\eta$ : Aut $F_{n} \rightarrow \operatorname{Hom}_{\mathbb{Q}}\left(\mathrm{gr}^{1} J, \mathrm{gr}^{2} J\right)$ such that the restriction of $\eta$ to $\mathcal{E}_{n}(1)$ is $\eta_{1}$.

At the present stage, we do not know whether the extension $\eta$ is unique up to 1-coboundary or not since we cannot determine the first cohomology group of Aut $F_{n}$ with coefficients in $\operatorname{Hom}_{\mathbb{Q}}\left(\mathrm{gr}^{1} J, \mathrm{gr}^{2} J\right)$ due to the combinatorial complexity. By using his extended crossed homomorphisms, Kawazumi [2006] constructed twisted higher cocycles of Aut $F_{n}$, and investigated its restriction to the mapping class group. In particular, he expressed the Morita-Mumford classes as a cup product of the twisted cocycles. In order to study the twisted cohomology groups of Aut $F_{n}$ with coefficients in modules of the Fricke characters, we are convinced that our work establishes a foothold as a first step.

In Section 2, we review the definitions of Fricke characters, the Johnson homomorphisms $\tau_{k}$ and the homomorphisms $\eta_{k}$. In Section 3, we extend the homomorphism $\eta_{1}$ to Aut $F_{n}$ as a crossed homomorphism. In Section 4, we show that the crossed homomorphism $\eta$ is not null cohomologous in the first cohomology group 
of Aut $F_{n}$ with coefficients in $\operatorname{Hom}_{\mathbb{Q}}\left(\mathrm{gr}^{1} J, \mathrm{gr}^{2} J\right)$. Furthermore, in Section 5, we calculate the image of Nielsen's generators of Aut $F_{n}$ by $\eta$.

\section{Preliminaries}

2A. Notation and conventions. Throughout the paper, we use the following notation and conventions: Let $G$ be a group and $N$ a normal subgroup of $G$.

- The abelianization of $G$ is denoted by $G^{\mathrm{ab}}$ unless otherwise noted.

- The automorphism group Aut $G$ of $G$ acts on $G$ from the right. For any $\sigma \in$ Aut $G$ and $x \in G$, the action of $\sigma$ on $x$ is denoted by $x^{\sigma}$.

- For an element $g \in G$, we also denote the coset class of $g$ in $G / N$ by $g$ if there is no confusion in context.

- For any elements $x$ and $y$ of $G$, the commutator bracket $[x, y]$ of $x$ and $y$ is defined to be $[x, y]:=x y x^{-1} y^{-1}$.

2B. The rings of Fricke characters. In this subsection, we review the rings of Fricke characters of the free group $F_{n}$. Let $R\left(F_{n}\right)$ be the set $\operatorname{Hom}\left(F_{n}, \operatorname{SL}(2, \mathbb{C})\right)$ of all $\operatorname{SL}(2, \mathbb{C})$-representations of $F_{n}$, and $\mathcal{F}\left(R\left(F_{n}\right), \mathbb{C}\right)$ the set of all complex-valued functions on $R\left(F_{n}\right)$. Then $\mathcal{F}\left(R\left(F_{n}\right), \mathbb{C}\right)$ naturally has a $\mathbb{C}$-algebra structure by the operations defined by

$$
\begin{aligned}
\left(\chi+\chi^{\prime}\right)(\rho) & :=\chi(\rho)+\chi^{\prime}(\rho), \\
\left(\chi \chi^{\prime}\right)(\rho) & :=\chi(\rho) \chi^{\prime}(\rho), \\
(\lambda \chi)(\rho) & :=\lambda(\chi(\rho)),
\end{aligned}
$$

for any $\chi, \chi^{\prime} \in \mathcal{F}\left(R\left(F_{n}\right), \mathbb{C}\right), \lambda \in \mathbb{C}$ and $\rho \in R\left(F_{n}\right)$. The group Aut $F_{n}$ naturally acts on $R\left(F_{n}\right)$ and $\mathcal{F}\left(R\left(F_{n}\right), \mathbb{C}\right)$ from the right by

$$
\begin{array}{ll}
\rho^{\sigma}(x):=\rho\left(x^{\sigma^{-1}}\right) & \text { for } \rho \in R\left(F_{n}\right) \text { and } x \in F_{n}, \\
\chi^{\sigma}(\rho):=\chi\left(\rho^{\sigma^{-1}}\right) & \text { for } \chi \in \mathcal{F}\left(R\left(F_{n}\right), \mathbb{C}\right) \text { and } \rho \in R\left(F_{n}\right),
\end{array}
$$

for any $\sigma \in$ Aut $F_{n}$. For any $x \in F_{n}$, we define an element $\operatorname{tr} x$ of $\mathcal{F}\left(R\left(F_{n}\right), \mathbb{C}\right)$ by

$$
(\operatorname{tr} x)(\rho):=\operatorname{tr} \rho(x)
$$

for any $\rho \in R\left(F_{n}\right)$. Here tr on the right-hand side means the trace of $2 \times 2$ matrix $\rho(x)$. The element $\operatorname{tr} x$ is called the Fricke character of $x \in F_{n}$. The action of an element $\sigma \in$ Aut $F_{n}$ on $\operatorname{tr} x$ is given by $\operatorname{tr} x^{\sigma}$. We have the following well-known formulae:
(1) $\operatorname{tr} x y+\operatorname{tr} x y^{-1}=(\operatorname{tr} x)(\operatorname{tr} y)$,
(2) $\operatorname{tr} x y z+\operatorname{tr} y x z=(\operatorname{tr} x)(\operatorname{tr} y z)+(\operatorname{tr} y)(\operatorname{tr} x z)+(\operatorname{tr} z)(\operatorname{tr} x y)-(\operatorname{tr} x)(\operatorname{tr} y)(\operatorname{tr} z)$,
(3) $\operatorname{tr}[x, y]=(\operatorname{tr} x)^{2}+(\operatorname{tr} y)^{2}+(\operatorname{tr} x y)^{2}-(\operatorname{tr} x)(\operatorname{tr} y)(\operatorname{tr} x y)-2$, 
(4)

$$
\begin{aligned}
2 \operatorname{tr} x y z w= & (\operatorname{tr} x)(\operatorname{tr} y z w)+(\operatorname{tr} y)(\operatorname{tr} z w x)+(\operatorname{tr} z)(\operatorname{tr} w x y)+(\operatorname{tr} w)(\operatorname{tr} x y z) \\
& +(\operatorname{tr} x y)(\operatorname{tr} z w)-(\operatorname{tr} x z)(\operatorname{tr} y w)+(\operatorname{tr} x w)(\operatorname{tr} y z) \\
& -(\operatorname{tr} x)(\operatorname{tr} y)(\operatorname{tr} z w)-(\operatorname{tr} y)(\operatorname{tr} z)(\operatorname{tr} x w)-(\operatorname{tr} x)(\operatorname{tr} w)(\operatorname{tr} y z) \\
& -(\operatorname{tr} z)(\operatorname{tr} w)(\operatorname{tr} x y)+(\operatorname{tr} x)(\operatorname{tr} y)(\operatorname{tr} z)(\operatorname{tr} w)
\end{aligned}
$$

for any $x, y, z, w \in F_{n}$. Equations (2) and (4) are due to Vogt [1889]. (For details, see [Maclachlan and Reid 2003, Section 3.4] for example.) The point of (2) is that $\operatorname{tr} y x z$ can be written as a sum of $-\operatorname{tr} x y z$ and a polynomial in $\operatorname{tr} v$, with $v$ a word in $x, y, z$ of length at most two. Similarly, the point of (4) is that tr $x y z w$ can be written as a polynomial in tr $v$ with $v$ a word in $x, y, z, w$ of length at most three.

Let $\mathfrak{X}_{\mathbb{Q}}\left(F_{n}\right)$ be the $\mathbb{Q}$-vector subspace of $\mathcal{F}\left(R\left(F_{n}\right), \mathbb{C}\right)$ generated by all tr $x$ for $x \in F_{n}$. From (1), it turns out that $\mathfrak{X}_{\mathbb{Q}}\left(F_{n}\right)$ is a $\mathbb{Q}$-subalgebra of $\mathcal{F}\left(R\left(F_{n}\right), \mathbb{C}\right)$. We call $\mathfrak{X}_{\mathbb{Q}}\left(F_{n}\right)$ the ring of Fricke characters of $F_{n}$ over $\mathbb{Q}$. Let $\mathbb{Q}[t]$ be the rational polynomial ring

$$
\mathbb{Q}\left[t_{i_{1} \cdots i_{l}} \mid 1 \leq l \leq 3,1 \leq i_{1}<i_{2}<\cdots<i_{l} \leq n\right]
$$

of $n+\left(\begin{array}{l}n \\ 2\end{array}\right)+\left(\begin{array}{l}n \\ 3\end{array}\right)$ indeterminates. Consider the ring homomorphism

$$
\pi: \mathbb{Q}[t] \rightarrow \mathcal{F}\left(R\left(F_{n}\right), \mathbb{C}\right)
$$

defined by

$$
\pi(1):=\frac{1}{2}\left(\operatorname{tr} 1_{F_{n}}\right), \quad \pi\left(t_{i_{1} \cdots i_{l}}\right):=\operatorname{tr} x_{i_{1}} \cdots x_{i_{l}} .
$$

Clearly, $\operatorname{Im} \pi \subset \mathfrak{X}_{\mathbb{Q}}\left(F_{n}\right)$. By a classical result due to Horowitz [1972], we have:

Theorem 2.1. For any $n \geq 2$, the homomorphism $\pi: \mathbb{Q}[t] \rightarrow \mathfrak{X}_{\mathbb{Q}}\left(F_{n}\right)$ is surjective.

More precisely, Horowitz obtained a generating set of the ring of Fricke characters of $G$ over $\mathbb{Z}$. Using this and (4), we can obtain the above theorem. Set

$$
I:=\operatorname{Ker} \pi=\left\{f \in \mathbb{Q}[t] \mid f\left(\operatorname{tr} \rho\left(x_{i_{1}} \cdots x_{i_{l}}\right)\right)=0 \text { for any } \rho \in R\left(F_{n}\right)\right\} .
$$

Then $\pi$ induces an isomorphism $\mathbb{Q}[t] / I \rightarrow \mathfrak{X}_{\mathbb{Q}}\left(F_{n}\right)$. In this paper, we always identify $\mathbb{Q}[t] / I$ with $\mathfrak{X}_{\mathbb{Q}}\left(F_{n}\right)$ through this isomorphism, and also call $\mathbb{Q}[t] / I$ the ring of Fricke characters of $F_{n}$ over $\mathbb{Q}$. We define an action of Aut $F_{n}$ on $\mathbb{Q}[t] / I$ such that the isomorphism $\mathbb{Q}[t] / I \stackrel{\cong}{\rightarrow} \mathfrak{X}_{\mathbb{Q}}\left(F_{n}\right)$ is Aut $F_{n}$-equivariant.

We remark that the structure of the ideal $I$ is quite complicated in general. It is an open problem to find a generating set for $I$ when $n \geq 4$. Horowitz [1972] showed that $I=(0)$ for $n=1$ and 2, and that $I$ is the principal ideal generated by a quadratic polynomial

$$
t_{123}^{2}-P_{123}(t) t_{123}+Q_{123}(t),
$$


where

$$
\begin{aligned}
P_{a b c}(t) & :=t_{a b} t_{c}+t_{a c} t_{b}+t_{b c} t_{a}-t_{a} t_{b} t_{c}, \\
Q_{a b c}(t) & :=t_{a}^{2}+t_{b}^{2}+t_{c}^{2}+t_{a b}^{2}+t_{a c}^{2}+t_{b c}^{2}-t_{a} t_{b} t_{a b}-t_{a} t_{c} t_{a c}-t_{b} t_{c} t_{b c}+t_{a b} t_{b c} t_{a c}-4 .
\end{aligned}
$$

For $n \geq 4$, Whittemore [1973] showed that the ideal $I$ is not principal. However, very little is known further in this case.

Although we can obtain a representation of Aut $F_{n}$ by the action of Aut $F_{n}$ on $\mathfrak{X}_{\mathbb{Q}}\left(F_{n}\right) \cong \mathbb{Q}[t] / I$, it is an infinite-degree representation, and hence it is not so easy to handle. In order to construct finite-dimensional representations of Aut $F_{n}$, we consider a descending filtration of Aut $F_{n}$-invariant ideals of $\mathbb{Q}[t] / I$, and take its graded quotients. Set $t_{i_{1} \cdots i_{l}}^{\prime}:=t_{i_{1} \cdots i_{l}}-2 \in \mathbb{Q}[t]$. For simplicity, we also denote by $t_{i_{1} \cdots i_{l}}^{\prime}$ its coset class in $\mathbb{Q}[t] / I$ by abuse of notation. Consider the ideal

$$
J:=\left(t_{i_{1} \cdots i_{l}}^{\prime} \mid 1 \leq l \leq 3,1 \leq i_{1}<i_{2}<\cdots<i_{l} \leq n\right) \subset \mathbb{Q}[t] / I
$$

of $\mathbb{Q}[t] / I$ generated by all $t_{i_{1} \cdots i_{l}}^{\prime}$ s. Then, we have a descending filtration

$$
J \supset J^{2} \supset J^{3} \supset \cdots
$$

of Aut $F_{n}$-invariant ideals of $\mathbb{Q}[t] / I$. Set $\operatorname{gr}^{k} J:=J^{k} / J^{k+1}$ for each $k \geq 1$. Then each $\mathrm{gr}^{k} J$ is an Aut $F_{n}$-invariant finite-dimensional $\mathbb{Q}$-vector space. In order to describe the action of Aut $F_{n}$ on $\mathrm{gr}^{k} J$ precisely, we have to find a basis of it. In general, however, by combinatorial complexities, it is quite a difficult problem. In [HS 2014], we explicitly gave bases of $\mathrm{gr}^{k} J$ for $k=1$ and 2 (see Section 4). For $k \geq 3$, even the dimension of $\mathrm{gr}^{k} J$ is not determined.

In [Hatakenaka and Satoh 2015], we studied the rings of Fricke characters of free abelian groups by a parallel argument as above. We review that work briefly. Let $H$ be the abelianization of $F_{n}$. The (coset classes of) $x_{1}, x_{2}, \ldots, x_{n}$ form a basis of $H$. Let $\mathfrak{X}_{\mathbb{Q}}(H)$ be the ring of Fricke characters of $H$ over $\mathbb{Q}$. Since

$$
2 \operatorname{tr} x y z=(\operatorname{tr} x)(\operatorname{tr} y z)+(\operatorname{tr} y)(\operatorname{tr} x z)+(\operatorname{tr} z)(\operatorname{tr} x y)-(\operatorname{tr} x)(\operatorname{tr} y)(\operatorname{tr} z)
$$

in $\mathfrak{X}_{\mathbb{Q}}(H)$ for any $x, y, z \in H$, by using Horowitz's result as mentioned above, we can see that $\mathfrak{X}_{\mathbb{Q}}(H)$ is generated by $\operatorname{tr} x_{i}$ for $1 \leq i \leq n$ and $\operatorname{tr} x_{i} x_{j}$ for $1 \leq i<j \leq n$. Let $J_{H}$ be the ideal generated by all $\operatorname{tr}^{\prime} x_{i}:=\operatorname{tr} x_{i}-2$ for $1 \leq i \leq n$ and $\operatorname{tr}^{\prime} x_{i} x_{j}:=\operatorname{tr} x_{i} x_{j}-2$ for $1 \leq i<j \leq n$. Then we have a descending filtration $J_{H} \supset J_{H}^{2} \supset \cdots$. We obtained a basis of the graded quotient $\mathrm{gr}^{k} J_{H}$ as a $\mathbb{Q}$-vector space for any $k \geq 1$ :

Theorem 2.2 [Hatakenaka and Satoh 2015]. For any $n \geq 2$ and $k \geq 1$,

$$
\begin{aligned}
& \bigcup_{l=0}^{k}\left\{\left(\operatorname{tr}^{\prime} x_{p_{1}} x_{q_{1}}\right) \cdots\left(\operatorname{tr}^{\prime} x_{p_{l}} x_{q_{l}}\right)\left(\operatorname{tr}^{\prime} x_{i_{l+1}}\right) \cdots\left(\operatorname{tr}^{\prime} x_{i_{k}}\right)\right. \\
& \left.\qquad \mid 1 \leq p_{1}<q_{1}<\cdots<p_{l}<q_{l} \leq n, 1 \leq i_{l+1} \leq \cdots \leq i_{k} \leq n\right\}
\end{aligned}
$$

is a basis of $\mathrm{gr}^{k} J_{H}$. 
As a corollary, we obtain a lower bound on the dimension of $\mathrm{gr}^{k} J$ by

$$
\operatorname{dim}_{\mathbb{Q}}\left(\operatorname{gr}^{k} J\right) \geq \operatorname{dim}_{\mathbb{Q}}\left(\operatorname{gr}^{k} J_{H}\right)=\sum_{l=0}^{k}\left(\begin{array}{c}
n \\
2 l
\end{array}\right)\left(\begin{array}{c}
n+k-l-1 \\
k-l
\end{array}\right)
$$

through the surjective homomorphism $\mathrm{gr}^{k} J \rightarrow \mathrm{gr}^{k} J_{H}$ induced from the abelianization $F_{n} \rightarrow H$.

2C. Johnson homomorphisms of Aut $\boldsymbol{F}_{\boldsymbol{n}}$. In this subsection, we review the Johnson homomorphisms of Aut $F_{n}$ (for details, see [Satoh 2013b], for example). Let $\rho:$ Aut $F_{n} \rightarrow$ Aut $H$ be the natural homomorphism induced from the abelianization of $F_{n}$. We identify Aut $H$ with the general linear group $\operatorname{GL}(n, \mathbb{Z})$ by fixing the basis $x_{1}, \ldots, x_{n}$ of $H$. The kernel IA If $_{n}$ of is called the IA-automorphism group of $F_{n}$. Let $\Gamma_{n}(1) \supset \Gamma_{n}(2) \supset \cdots$ be the lower central series of a free group $F_{n}$ defined by the rule

$$
\Gamma_{n}(1):=F_{n}, \quad \Gamma_{n}(k):=\left[\Gamma_{n}(k-1), F_{n}\right], \quad k \geq 2 .
$$

We denote by $\mathcal{L}_{n}(k):=\Gamma_{n}(k) / \Gamma_{n}(k+1)$ the graded quotient of the lower central series of $F_{n}$. For each $k \geq 1$, the natural action of Aut $F_{n}$ on $\mathcal{L}_{n}(k)$ induces that of $\mathrm{GL}(n, \mathbb{Z})$ since $\mathrm{IA}_{n}$ acts on $\mathcal{L}_{n}(k)$ trivially.

For each $k \geq 1$, the action of Aut $F_{n}$ on the nilpotent quotient group $F_{n} / \Gamma_{n}(k+1)$ induces a homomorphism

$$
\text { Aut } F_{n} \rightarrow \operatorname{Aut}\left(F_{n} / \Gamma_{n}(k+1)\right) \text {. }
$$

We denote its kernel by $\mathcal{A}_{n}(k)$. Then the groups $\mathcal{A}_{n}(k)$ define a descending central filtration

$$
\mathrm{IA}_{n}=\mathcal{A}_{n}(1) \supset \mathcal{A}_{n}(2) \supset \mathcal{A}_{n}(3) \supset \cdots
$$

of $\mathrm{IA}_{n}$. We call this the Andreadakis-Johnson filtration of Aut $F_{n}$; historically, it was introduced by Andreadakis [1965], who showed:

Theorem 2.3. (1) For any $k, l \geq 1, \sigma \in \mathcal{A}_{n}(k)$ and $x \in \Gamma_{n}(l), x^{-1} x^{\sigma} \in \Gamma_{n}(k+l)$.

(2) For any $k$ and $l \geq 1,\left[\mathcal{A}_{n}(k), \mathcal{A}_{n}(l)\right] \subset \mathcal{A}_{n}(k+l)$.

(3) $\bigcap_{k \geq 1} \mathcal{A}_{n}(k)=1$.

In the 1980s, Dennis Johnson studied this type of filtration for the mapping class group of a surface, and this became known as the Johnson filtration of the mapping class group.

For each $k \geq 1$, the group Aut $F_{n}$ acts on $\mathcal{A}_{n}(k)$ by conjugation, and it naturally induces an action of $\mathrm{GL}(n, \mathbb{Z})=$ Aut $F_{n} / \mathrm{IA}_{n}$ on the graded quotients $\mathrm{gr}^{k} \mathcal{A}_{n}:=$ $\mathcal{A}_{n}(k) / \mathcal{A}_{n}(k+1)$ by Theorem $2.3(2)$. In order to study the $\mathrm{GL}(n, \mathbb{Z})$-module 
structure of $\mathrm{gr}^{k} \mathcal{A}_{n}$, we consider the Johnson homomorphisms of Aut $F_{n}$. For each $k \geq 1$, define a homomorphism $\tilde{\tau}_{k}: \mathcal{A}_{n}(k) \rightarrow \operatorname{Hom}_{\mathbb{Z}}\left(H, \mathcal{L}_{n}(k+1)\right)$ by

$$
\sigma \mapsto\left(x \mapsto x^{-1} x^{\sigma}\right), \quad x \in H .
$$

Then the kernel of $\tilde{\tau}_{k}$ is just $\mathcal{A}_{n}(k+1)$. Hence it induces an injective homomorphism

$$
\tau_{k}: \operatorname{gr}^{k} \mathcal{A}_{n} \hookrightarrow \operatorname{Hom}_{\mathbb{Z}}\left(H, \mathcal{L}_{n}(k+1)\right)=H^{*} \otimes_{\mathbb{Z}} \mathcal{L}_{n}(k+1) .
$$

The homomorphisms $\tilde{\tau}_{k}$ and $\tau_{k}$ are called the $k$-th Johnson homomorphisms of Aut $F_{n}$. We can see each $\tau_{k}$ is $\operatorname{GL}(n, \mathbb{Z})$-equivariant.

Now, we have a question to ask whether the first Johnson homomorphism can be extended to Aut $F_{n}$ or not. Such study and results were given by Morita [1993b] for the mapping class group of a surface, and by Kawazumi [2006] for the automorphism group of a free group as mentioned in the introduction. In particular, Kawazumi showed the first rational Johnson homomorphism

$$
\tilde{\tau}_{1}: \mathrm{IA}_{n} \rightarrow H^{*} \otimes_{\mathbb{Z}} \Lambda^{2} H \rightarrow\left(H^{*} \otimes_{\mathbb{Z}} \Lambda^{2} H\right) \otimes_{\mathbb{Z}} \mathbb{Q}
$$

can be extended to Aut $F_{n}$ as a crossed homomorphism. In [Satoh 2013a], we showed that

$$
H^{1}\left(\text { Aut } F_{n},\left(H^{*} \otimes_{\mathbb{Z}} \Lambda^{2} H\right) \otimes_{\mathbb{Z}} \mathbb{Q}\right) \cong \mathbb{Q}^{\oplus 2},
$$

and that the extension of the first Johnson homomorphism and a crossed homomorphism obtained from the Magnus representation form a basis of the above first cohomology group. These results are free group analogues of Morita's work [1993b] for the mapping class group of a surface.

2D. Homomorphisms $\eta_{\boldsymbol{k}}$. In [HS 2014], we introduced homomorphisms $\eta_{k}$ which are Fricke character analogue of the Johnson homomorphisms $\tau_{k}$. Here we review the definition of $\eta_{k}$.

For any $k \geq 1$, let

$$
\mathcal{E}_{n}(k):=\operatorname{Ker}\left(\text { Aut } F_{n} \rightarrow \operatorname{Aut}\left(J / J^{k+1}\right)\right)
$$

be the kernel of a homomorphism Aut $F_{n} \rightarrow \operatorname{Aut}\left(J / J^{k+1}\right)$ induced from the action of Aut $F_{n}$ on $J / J^{k+1}$. Then the groups $\mathcal{E}_{n}(k)$ define a descending filtration

$$
\mathcal{E}_{n}(1) \supset \mathcal{E}_{n}(2) \supset \cdots \supset \mathcal{E}_{n}(k) \supset \cdots
$$

of Aut $F_{n}$.

Theorem 2.4 [HS 2014]. For any $n \geq 3$, we have:

(1) For any $k, l \geq 1,\left[\mathcal{E}_{n}(k), \mathcal{E}_{n}(l)\right] \subset \mathcal{E}_{n}(k+l)$.

(2) $\mathcal{E}_{n}(1)=\operatorname{Inn} F_{n} \cdot \mathcal{A}_{n}(2)$, where $\operatorname{Inn} F_{n}$ is the inner automorphism group of $F_{n}$.

(3) For any $k \geq 1, \mathcal{A}_{n}(2 k) \subset \mathcal{E}_{n}(k)$. 
From Theorem 2.4(1), the graded quotients $\operatorname{gr}^{k} \mathcal{E}_{n}:=\mathcal{E}_{n}(k) / \mathcal{E}_{n}(k+1)$ are abelian groups for any $k \geq 1$. Since each $\mathcal{E}_{n}(k)$ is a normal subgroup of Aut $F_{n}$, the group Aut $F_{n}$ naturally acts on $\mathrm{gr}^{k} \mathcal{E}_{n}$ by the conjugation from the right. Namely, for any $\sigma \in$ Aut $F_{n}$ and $\tau \in \mathcal{E}_{n}(k)$, the action of $\sigma$ on $\tau\left(\bmod \mathcal{E}_{n}(k+1)\right)$ is given by

$$
\left(\tau\left(\bmod \mathcal{E}_{n}(k+1)\right)\right) \cdot \sigma:=\sigma^{-1} \tau \sigma\left(\bmod \mathcal{E}_{n}(k+1)\right) .
$$

Furthermore, since $\left\{\mathcal{E}_{n}(k)\right\}$ is a central filtration, the action of $\mathcal{E}_{n}(1)$ on $\mathrm{gr}^{k} \mathcal{E}_{n}$ is trivial. Hence we can consider each $\operatorname{gr}^{k} \mathcal{E}_{n}$ as an Aut $F_{n} / \mathcal{E}_{n}(1)$-module. In order to investigate the Aut $F_{n} / \mathcal{E}_{n}(1)$-module structure of $\operatorname{gr}^{k} \mathcal{E}_{n}$, we have introduced

$$
\eta_{k}: \mathrm{gr}^{k} \mathcal{E}_{n} \rightarrow \operatorname{Hom}_{\mathbb{Q}}\left(\mathrm{gr}^{1} J, \mathrm{gr}^{k+1} J\right)
$$

defined by

$$
\sigma\left(\bmod \mathcal{E}_{n}(k+1)\right) \mapsto\left(f\left(\bmod J^{2}\right) \mapsto f^{\sigma}-f\left(\bmod J^{k+1}\right)\right) .
$$

In [HS 2014], we showed that each $\eta_{k}$ is an Aut $F_{n} / \mathcal{E}_{n}(1)$-equivariant injective homomorphism. However, the structure of the image of $\eta_{k}$ is not well-understood even in the case where $k=1$.

\section{An extension of $\eta_{1}$ as a crossed homomorphism}

In this section, we extend the homomorphism

$$
\tilde{\eta}_{1}: \mathcal{E}_{n}(1) \rightarrow \mathrm{gr}^{1} \mathcal{E}_{n} \stackrel{\eta_{1}}{\rightarrow} \operatorname{Hom}_{\mathbb{Q}}\left(\mathrm{gr}^{1} J, \mathrm{gr}^{2} J\right)
$$

to Aut $F_{n}$ as a crossed homomorphism, following [Morita 1993b]. Furthermore, according to the usual convention in homological algebra, for any group $G$ and $G$-module $M$, we consider that $G$ acts on $M$ from the left unless otherwise noted. Hence, the right actions mentioned above are read as left actions in the natural way. For example, for any $\sigma \in$ Aut $F_{n}$ and $x \in F_{n}$, the left action of $\sigma$ on the Fricke character tr $x$ is given by

$$
\sigma \cdot(\operatorname{tr} x)=\operatorname{tr} x^{\sigma^{-1}}
$$

For basic materials for cohomology of associative algebras, see [Cartan and Eilenberg 1999, Chapter IX], for example.

First, consider an extension

$$
0 \rightarrow J^{2} / J^{3} \rightarrow \mathfrak{X}_{\mathbb{Q}}\left(F_{n}\right) / J^{3} \rightarrow \mathfrak{X}_{\mathbb{Q}}\left(F_{n}\right) / J^{2} \rightarrow 0
$$

of associative $\mathbb{Q}$-algebras. For an associative ring $R$, we denote by $\operatorname{Aut}_{(\mathrm{Ring})}(R)$ the ring automorphism group of $R$. For a $\mathbb{Q}$-vector space $M$, we denote by $\operatorname{Aut}(M)$ the $\mathbb{Q}$-linear automorphism group of $M$. 


\section{Proposition 3.1. The natural homomorphism}

$$
\Phi: \operatorname{Aut}_{(\text {Ring })}\left(\mathfrak{X}_{\mathbb{Q}}\left(F_{n}\right) / J^{3}\right) \rightarrow \operatorname{Aut}_{(\text {Ring })}\left(\mathfrak{X}_{\mathbb{Q}}\left(F_{n}\right) / J^{2}\right)
$$

is surjective.

Proof. First, observe the characteristic class $\theta\left(\mathrm{id}_{J / J^{2}}\right)$ of the extension (5), where

$$
\theta: \operatorname{Hom}_{E\left(\mathfrak{X}_{\mathbb{Q}}\left(F_{n}\right) / J^{2}\right)}\left(J^{2} / J^{3}, J^{2} / J^{3}\right) \rightarrow H^{2}\left(\mathfrak{X}_{\mathbb{Q}}\left(F_{n}\right) / J^{2}, J^{2} / J^{3}\right)
$$

is the connecting homomorphism, and $E\left(\mathfrak{X}_{\mathbb{Q}}\left(F_{n}\right) / J^{2}\right)$ is the enveloping algebra of $\mathfrak{X}_{\mathbb{Q}}\left(F_{n}\right) / J^{2}$. Choose a 2-cocycle $c$ of the algebra $\mathfrak{X}_{\mathbb{Q}}\left(F_{n}\right) / J^{2}$ with coefficients in $J^{2} / J^{3}$, which represents the cohomology class $\theta\left(\operatorname{id}_{J / J^{2}}\right)$. Then $\mathfrak{X}_{\mathbb{Q}}\left(F_{n}\right) / J^{3}$ can be explicitly described as the product $J^{2} / J^{3} \times \mathfrak{X}_{\mathbb{Q}}\left(F_{n}\right) / J^{2}$ equipped with the multiplication given by

$$
(\xi, \tau)\left(\xi^{\prime}, \tau^{\prime}\right)=\left(\xi \tau^{\prime}+\tau \xi^{\prime}+c\left(\tau, \tau^{\prime}\right), \tau \tau^{\prime}\right)
$$

for any $\xi, \xi^{\prime} \in J^{2} / J^{3}$ and $\tau, \tau^{\prime} \in \mathfrak{X}_{\mathbb{Q}}\left(F_{n}\right) / J^{2}$. We denote by $\Lambda_{c}$ this associative algebra.

For any $\alpha \in \operatorname{Aut}_{(\operatorname{Ring})}\left(\mathfrak{X}_{\mathbb{Q}}\left(F_{n}\right) / J^{2}\right)$, since the cohomology class of $c$ is the characteristic class induced from id $J_{J / J^{2}}$, the 2-cocycle $\alpha^{\sharp}(c)$ should be cohomologous to $c$ where $\alpha^{\sharp}$ is the induced homomorphism from $\alpha$. Hence there exists a 1-chain $d: \mathfrak{X}_{\mathbb{Q}}\left(F_{n}\right) / J^{2} \rightarrow J^{2} / J^{3}$ such that $\alpha^{\sharp}(c)-c=\delta d$, where $\delta$ is the coboundary homomorphism. Namely, we have

$$
\alpha^{-1} \cdot c\left(\alpha(\tau), \alpha\left(\tau^{\prime}\right)\right)-c\left(\tau, \tau^{\prime}\right)=\tau d\left(\tau^{\prime}\right)-d\left(\tau \tau^{\prime}\right)+d(\tau) \tau^{\prime}
$$

for any $\tau, \tau^{\prime} \in \mathfrak{X}_{\mathbb{Q}}\left(F_{n}\right) / J^{2}$. Define the map $\tilde{\alpha}: \Lambda_{c} \rightarrow \Lambda_{c}$ to be

$$
(\xi, \tau) \mapsto(\alpha(\xi)-\alpha(d(\tau)), \alpha(\tau)) .
$$

Then $\tilde{\alpha} \in \operatorname{Aut}_{(\operatorname{Ring})}\left(\mathfrak{X}_{\mathbb{Q}}\left(F_{n}\right) / J^{3}\right)$ under the identification $\Lambda_{c}=\mathfrak{X}_{\mathbb{Q}}\left(F_{n}\right) / J^{3}$, and $\Phi(\tilde{\alpha})=\alpha$.

For any $f \in J$, we denote the coset class of $f$ in $J / J^{k}$ by $[f]_{k}$. For any $k \geq 2$, set

$$
\overline{\operatorname{Aut}}\left(J / J^{k}\right):=\left\{\sigma \in \operatorname{Aut}\left(J / J^{k}\right) \mid \sigma\left(\left[\gamma \gamma^{\prime}\right]_{k}\right)=\sigma\left([\gamma]_{k}\right) \sigma\left(\left[\gamma^{\prime}\right]_{k}\right), \gamma, \gamma^{\prime} \in J\right\} .
$$

Note that $\overline{\operatorname{Aut}}\left(J / J^{2}\right)=\operatorname{Aut}\left(J / J^{2}\right)=\operatorname{GL}_{\mathbb{Q}}\left(J / J^{2}\right)$.

Lemma 3.2. The group homomorphism

$$
\Psi: \operatorname{Aut}_{(\operatorname{Ring})}\left(\mathfrak{X}_{\mathbb{Q}}\left(F_{n}\right) / J^{k}\right) \rightarrow \overline{\operatorname{Aut}}\left(J / J^{k}\right),
$$

defined by $\left.\sigma \mapsto \sigma\right|_{J / J^{k}}$, is an isomorphism. 
Proof. Consider the polynomial ring

$$
\mathbb{Q}\left[t^{\prime}\right]:=\mathbb{Q}\left[t_{i_{1} \cdots i_{l}}^{\prime} \mid 1 \leq l \leq 3,1 \leq i_{1}<i_{2}<\cdots<i_{l} \leq n\right]
$$

with indeterminates $t_{i_{1} \cdots i_{l}}^{\prime}$, and the natural surjection $\pi^{\prime}: \mathbb{Q}\left[t^{\prime}\right] \rightarrow \mathfrak{X}_{\mathbb{Q}}\left(F_{n}\right)$ given by $1 \mapsto \frac{1}{2}\left(\operatorname{tr} 1_{F_{n}}\right)$ and $t_{i_{1} \cdots i_{l}}^{\prime} \mapsto \operatorname{tr}^{\prime} x_{i_{1}} \cdots x_{i_{l}}$. The kernel $I^{\prime}$ of $\pi^{\prime}$ is contained in the ideal $J^{\prime}$ generated by all $t_{i_{1} \cdots i_{l}}^{\prime}$.

We construct the inverse map of $\Psi$. For any $\beta \in \overline{\operatorname{Aut}}\left(J / J^{k}\right)$, define the $\mathbb{Q}$-algebra homomorphism $\tilde{\beta}: \mathbb{Q}\left[t^{\prime}\right] \rightarrow \mathfrak{X}_{\mathbb{Q}}\left(F_{n}\right) / J^{k}$ to be

$$
\tilde{\beta}(1):=\left[\frac{1}{2}\left(\operatorname{tr} 1_{F_{n}}\right)\right]_{k}, \quad \tilde{\beta}\left(t_{i_{1} \cdots i_{l}}^{\prime}\right):=\beta\left(\left[\pi^{\prime}\left(t_{i_{1} \cdots i_{l}}^{\prime}\right)\right]_{k}\right) .
$$

Since $I^{\prime} \subset \operatorname{Ker} \tilde{\beta}$ and $\tilde{\beta}\left(J^{\prime}\right)=J$, the above $\tilde{\beta}$ induces a ring homomorphism $\mathfrak{X}_{\mathbb{Q}}\left(F_{n}\right) / J^{k} \rightarrow \mathfrak{X}_{\mathbb{Q}}\left(F_{n}\right) / J^{k}$, say $\tilde{\beta}$, by abuse of notation. Since $\beta$ is an automorphism, so is $\tilde{\beta}$. Then we have the homomorphism $\Psi^{\prime}: \overline{\operatorname{Aut}}\left(J / J^{k}\right) \rightarrow \operatorname{Aut}\left(\mathfrak{X}_{\mathbb{Q}}\left(F_{n}\right) / J^{k}\right)$ defined by $\beta \mapsto \tilde{\beta}$, and see that $\Psi^{\prime}$ is the inverse of $\Psi$.

From Proposition 3.1 and Lemma 3.2, we obtain the induced surjective homomorphism

$$
\varphi: \overline{\operatorname{Aut}}\left(J / J^{3}\right) \rightarrow \overline{\operatorname{Aut}}\left(J / J^{2}\right) .
$$

Next, we consider an embedding of $\operatorname{Hom}_{\mathbb{Q}}\left(\operatorname{gr}^{1} J, \operatorname{gr}^{2} J\right)$ into $\overline{\operatorname{Aut}}\left(J / J^{3}\right)$. For any $f \in \operatorname{Hom}_{\mathbb{Q}}\left(\mathrm{gr}^{1} J, \mathrm{gr}^{2} J\right)$, define the map $\tilde{f}: J / J^{3} \rightarrow J / J^{3}$ by

$$
\tilde{f}\left([\gamma]_{3}\right):=[\gamma]_{3}+f\left([\gamma]_{2}\right)
$$

for any $\gamma \in J$.

Proposition 3.3. With the above notation, for any $f \in \mathrm{Hom}_{\mathbb{Q}}\left(\mathrm{gr}^{1} J, \mathrm{gr}^{2} J\right)$, we see $\tilde{f} \in \overline{\operatorname{Aut}}\left(J / J^{3}\right)$, and the map

$$
\iota: \operatorname{Hom}_{\mathbb{Q}}\left(\operatorname{gr}^{1} J, \operatorname{gr}^{2} J\right) \rightarrow \overline{\operatorname{Aut}}\left(J / J^{3}\right),
$$

defined by $f \mapsto \tilde{f}$, is injective.

Proof. First, we show $\tilde{f}$ is a homomorphism. For any $\gamma, \gamma^{\prime} \in J$,

$$
\begin{aligned}
\tilde{f}\left([\gamma]_{3}+\left[\gamma^{\prime}\right]_{3}\right) & =\tilde{f}\left(\left[\gamma+\gamma^{\prime}\right]_{3}\right)=\left[\gamma+\gamma^{\prime}\right]_{3}+f\left(\left[\gamma+\gamma^{\prime}\right]_{2}\right) \\
& =\left([\gamma]_{3}+f\left([\gamma]_{2}\right)\right)+\left(\left[\gamma^{\prime}\right]_{3}+f\left(\left[\gamma^{\prime}\right]_{2}\right)\right) \\
& =\tilde{f}\left([\gamma]_{3}\right)+\tilde{f}\left(\left[\gamma^{\prime}\right]_{3}\right) .
\end{aligned}
$$

Thus $\tilde{f}$ is a homomorphism. Furthermore, $\tilde{f}$ satisfies

$$
\begin{aligned}
\tilde{f}\left([\gamma]_{3}\left[\gamma^{\prime}\right]_{3}\right) & =\tilde{f}\left(\left[\gamma \gamma^{\prime}\right]_{3}\right)=\left[\gamma \gamma^{\prime}\right]_{3}+f\left(\left[\gamma \gamma^{\prime}\right]_{2}\right)=[\gamma]_{3}\left[\gamma^{\prime}\right]_{3} \\
& =\left([\gamma]_{3}+f\left([\gamma]_{2}\right)\right)\left(\left[\gamma^{\prime}\right]_{3}+f\left(\left[\gamma^{\prime}\right]_{2}\right)\right)=\tilde{f}\left([\gamma]_{3}\right) \tilde{f}\left(\left[\gamma^{\prime}\right]_{3}\right)
\end{aligned}
$$


for any $\gamma, \gamma^{\prime} \in J$. On the other hand, we have $\widetilde{f+g}=\tilde{f} \circ \tilde{g}$ for any $f, g \in$ $\operatorname{Hom}_{\mathbb{Q}}\left(\mathrm{gr}^{1} J, \mathrm{gr}^{2} J\right)$. In fact, for any $\gamma \in J$,

$$
\begin{aligned}
& (\widetilde{f+g})\left([\gamma]_{3}\right)=[\gamma]_{3}+f\left([\gamma]_{2}\right)+g\left([\gamma]_{2}\right), \\
& (\tilde{f} \circ \tilde{g})\left([\gamma]_{3}\right)=\tilde{f}\left([\gamma]_{3}+g\left([\gamma]_{2}\right)\right)=[\gamma]_{3}+g\left([\gamma]_{2}\right)+f\left([\gamma]_{2}\right) .
\end{aligned}
$$

This shows that $\iota$ is a homomorphism. On the other hand, for the zero map $0 \in \operatorname{Hom}_{\mathbb{Q}}\left(\mathrm{gr}^{1} J, \mathrm{gr}^{2} J\right)$, it is obvious that $\tilde{0}=\mathrm{id}_{J / J^{3}}$. Hence each $\tilde{f}$ has its inverse map $\tilde{f}^{-1}=\widetilde{-f}$. This means $\tilde{f}$ an automorphism on $J / J^{3}$.

Finally, we show that $\iota$ is injective. Assume that $\tilde{f}=\mathrm{id}_{J / J^{3}}$ for some $f \in$ $\operatorname{Hom}_{\mathbb{Q}}\left(\operatorname{gr}^{1} J, \operatorname{gr}^{2} J\right)$. Then for any $[\gamma]_{3} \in J / J^{3}$, we have

$$
\tilde{f}\left([\gamma]_{3}\right)=[\gamma]_{3}+f\left([\gamma]_{2}\right)=[\gamma]_{3} .
$$

Hence $f\left([\gamma]_{2}\right)=0$ for any $[\gamma]_{2} \in J / J^{2}$, and $f=0$. This shows $\iota$ is injective.

Proposition 3.4. The sequence

$$
0 \rightarrow \operatorname{Hom}_{\mathbb{Q}}\left(\mathrm{gr}^{1} J, \operatorname{gr}^{2} J\right) \stackrel{\iota}{\rightarrow} \overline{\operatorname{Aut}}\left(J / J^{3}\right) \stackrel{\varphi}{\rightarrow} \overline{\operatorname{Aut}}\left(J / J^{2}\right) \rightarrow 1
$$

is a split group extension.

Proof. First, we show the above sequence is exact. Namely, it suffices to show $\operatorname{Im} \iota=\operatorname{Ker} \varphi$. The fact that $\operatorname{Im} \iota \subset \operatorname{Ker} \varphi$ follows from

$$
\begin{aligned}
(\varphi \circ \iota)(f)\left([\gamma]_{2}\right) & =\varphi(\tilde{f})\left([\gamma]_{2}\right)=[\gamma]_{3}+f\left([\gamma]_{2}\right)\left(\bmod J^{2}\right) \\
& =[\gamma]_{2}
\end{aligned}
$$

for any $f \in \operatorname{Hom}_{\mathbb{Q}}\left(\operatorname{gr}^{1} J, \operatorname{gr}^{2} J\right)$ and $\gamma \in J$. To show $\operatorname{Im} \iota \supset \operatorname{Ker} \varphi$, take any $f \in \operatorname{Ker} \varphi$. Define $g \in \operatorname{Hom}_{\mathbb{Q}}\left(\mathrm{gr}^{1} J, \mathrm{gr}^{2} J\right)$ to be

$$
g\left([\gamma]_{2}\right):=f\left([\gamma]_{3}\right)-[\gamma]_{3}
$$

for any $\gamma \in J$. The map $g$ is well-defined. In fact, for any $\gamma, \gamma^{\prime} \in J$ such that $[\gamma]_{2}=\left[\gamma^{\prime}\right]_{2}$, if we set $\gamma^{\prime}-\gamma=\varepsilon \in J^{2}$, then we have

$$
\begin{aligned}
g\left(\left[\gamma^{\prime}\right]_{2}\right) & =f\left(\left[\gamma^{\prime}\right]_{3}\right)-\left[\gamma^{\prime}\right]_{3}=f\left([\gamma+\varepsilon]_{3}\right)-[\gamma+\varepsilon]_{3} \\
& =f\left([\gamma]_{3}\right)-[\gamma]_{3}+f\left([\varepsilon]_{3}\right)-[\varepsilon]_{3}=f\left([\gamma]_{3}\right)-[\gamma]_{3} \\
& =g\left([\gamma]_{2}\right) .
\end{aligned}
$$

Here we remark that $f\left([\varepsilon]_{3}\right)-[\varepsilon]_{3}=0 \in J^{2} / J^{3}$ since $\varepsilon \in J^{2}$ and $f \in \operatorname{Ker} \varphi$. It is easy to show that $g$ is a homomorphism. Furthermore, for any $\gamma \in J$,

$$
\tilde{g}\left([\gamma]_{3}\right)=[\gamma]_{3}+g\left([\gamma]_{2}\right)=f\left([\gamma]_{3}\right) .
$$

This shows $f=\tilde{g}=\iota(g) \in \operatorname{Im} \iota$. 
Finally, we construct the section $s: \overline{\operatorname{Aut}}\left(J / J^{2}\right) \rightarrow \overline{\operatorname{Aut}}\left(J / J^{3}\right)$ of (6). Take elements $\gamma_{1}, \gamma_{2}, \ldots, \gamma_{p} \in J, \gamma_{p+1}, \ldots, \gamma_{p+q} \in J^{2}$ such that $\left(\left[\gamma_{1}\right]_{2},\left[\gamma_{2}\right]_{2}, \ldots,\left[\gamma_{p}\right]_{2}\right)$ and $\left(\left[\gamma_{p+1}\right]_{3}, \ldots,\left[\gamma_{p+q}\right]_{3}\right)$ form bases of $\operatorname{gr}^{1} J$ and $\operatorname{gr}^{2} J$, respectively. Then $\left(\left[\gamma_{1}\right]_{3},\left[\gamma_{2}\right]_{3}, \ldots,\left[\gamma_{p+q}\right]_{3}\right)$ is a basis of $J / J^{3}$.

For any $\beta \in \overline{\operatorname{Aut}}\left(J / J^{2}\right)$, there exists an element $\tilde{\beta} \in \overline{\operatorname{Aut}}\left(J / J^{3}\right)$ such that $\varphi(\tilde{\beta})=$ $\beta$. In general, for any $1 \leq j \leq p$, the image $\tilde{\beta}\left(\left[\gamma_{j}\right]_{3}\right)$ can be written as

$$
\tilde{\beta}\left(\left[\gamma_{j}\right]_{3}\right)=a_{1 j}\left[\gamma_{1}\right]_{3}+\cdots+a_{p j}\left[\gamma_{p}\right]_{3}+a_{p+1, j}\left[\gamma_{p+1}\right]_{3}+\cdots+a_{p+q, j}\left[\gamma_{p+q}\right]_{3}
$$

for some $a_{i j} \in \mathbb{Q}$. Since $\beta \in \operatorname{Aut}\left(J / J^{2}\right)$, if for any $1 \leq j \leq p$ we set

$$
v_{j}:=a_{1 j}\left[\gamma_{1}\right]_{2}+\cdots+a_{p j}\left[\gamma_{p}\right]_{2}
$$

then $\left(v_{1}, v_{2}, \ldots, v_{p}\right)$ is a basis of $\operatorname{gr}^{1} J$. Let $\delta=\delta_{\tilde{\beta}}: \mathrm{gr}^{1} J \rightarrow \mathrm{gr}^{2} J$ be the $\mathbb{Q}$-linear map given by

$$
\delta\left(v_{j}\right)=-\left(a_{p+1, j}\left[\gamma_{p+1}\right]_{3}+\cdots+a_{p+q, j}\left[\gamma_{p+q}\right]_{3}\right)
$$

for any $1 \leq j \leq p$. Then we obtain

$$
\begin{aligned}
(\tilde{\delta} \circ \tilde{\beta}) & \left(\left[\gamma_{j}\right]_{3}\right) \\
& =\tilde{\delta}\left(a_{1 j}\left[\gamma_{1}\right]_{3}+\cdots+a_{p j}\left[\gamma_{p}\right]_{3}+a_{p+1, j}\left[\gamma_{p+1}\right]_{3}+\cdots+a_{p+q, j}\left[\gamma_{p+q}\right]_{3}\right) \\
& =a_{1 j}\left[\gamma_{1}\right]_{3}+\cdots+a_{p j}\left[\gamma_{p}\right]_{3}+a_{p+1, j}\left[\gamma_{p+1}\right]_{3}+\cdots+a_{p+q, j}\left[\gamma_{p+q}\right]_{3}+\delta\left(v_{j}\right) \\
& =a_{1 j}\left[\gamma_{1}\right]_{3}+\cdots+a_{p j}\left[\gamma_{p}\right]_{3}
\end{aligned}
$$

for any $1 \leq j \leq p$. Consider the map $s: \overline{\operatorname{Aut}}\left(J / J^{2}\right) \rightarrow \overline{\operatorname{Aut}}\left(J / J^{3}\right)$ defined by $\beta \mapsto \tilde{\delta} \circ \tilde{\beta}$. We can see that $s$ is a homomorphism and is the required section. Hence the exact sequence (6) splits.

Now, we construct a crossed homomorphism of Aut $F_{n}$ which is an extension of $\tilde{\eta}_{1}$. We provide an easy exercise:

\section{Lemma 3.5. Let}

$$
0 \rightarrow K \rightarrow G \rightarrow N \rightarrow 1
$$

be a split extension of groups over $N$ with an additive abelian group $K$. For any $g$, there exist unique elements $k_{g} \in K$ and $n_{g} \in N$ such that $g=k_{g} n_{g}$. Then the map $k: G \rightarrow K$ defined by $g \mapsto k_{g}$ is a crossed homomorphism.

By using the above lemma, we obtain:

Theorem 3.6. There is a crossed homomorphism $\eta$ : Aut $F_{n} \rightarrow \operatorname{Hom}_{\mathbb{Q}}\left(\mathrm{gr}^{1} J, \mathrm{gr}^{2} J\right)$ such that the restriction of $\eta$ to $\mathcal{E}_{n}(1)$ is $\tilde{\eta}_{1}$. 
Proof. By applying Lemma 3.5 to the split extension (6), we obtain a crossed homomorphism $k: \overline{\operatorname{Aut}}\left(J / J^{3}\right) \rightarrow \operatorname{Hom}_{\mathbb{Q}}\left(\mathrm{gr}^{1} J, \mathrm{gr}^{2} J\right)$. Hence by composing $k$ and the natural homomorphism Aut $F_{n} \rightarrow \operatorname{Aut}\left(J / J^{3}\right)$, we obtain a crossed homomorphism

$$
\eta: \text { Aut } F_{n} \rightarrow \operatorname{Hom}_{\mathbb{Q}}\left(\mathrm{gr}^{1} J, \mathrm{gr}^{2} J\right) \text {. }
$$

This is the required homomorphism.

\section{Nontriviality of $\eta$ as a 1-cocycle}

In this section, we give a few remarks about the image of $\eta_{1}$ and the nontriviality of $\eta$ as a 1-cocycle.

The first Johnson homomorphism $\tau_{1}: \operatorname{gr}^{1} \mathcal{A}_{n} \rightarrow H^{*} \otimes_{\mathbb{Z}} \mathcal{L}_{n}(l+1)$ is surjective, and hence an isomorphism. We can easily see this fact by calculating the images of Magnus's generators of $\mathrm{IA}_{n}$, given by

$$
K_{i j}:\left\{\begin{array}{l}
x_{i} \mapsto x_{j}^{-1} x_{i} x_{j}, \\
x_{t} \mapsto x_{t} \quad(t \neq i),
\end{array}\right.
$$

for distinct $i, j \in\{1,2, \ldots, n\}$, and

$$
K_{i j k}:\left\{\begin{array}{l}
x_{i} \mapsto x_{i} x_{j} x_{k} x_{j}^{-1} x_{k}^{-1}, \\
x_{t} \mapsto x_{t} \quad(t \neq i),
\end{array}\right.
$$

for distinct $i, j, k \in\{1,2, \ldots, n\}$ such that $j<k$. From a viewpoint of a comparative study, it is a natural problem for us to determine the image of $\eta_{1}$. However, this is complicated for two reasons. One is that we do not have any generating set of $\mathcal{E}_{n}(1)$. From our result $\mathcal{E}_{n}(1)=\operatorname{Inn} F_{n} \cdot \mathcal{A}_{n}(2)$, it suffices to obtain a generating set of $\mathcal{A}_{n}(2)$. This seems, however, quite difficult in general. The other is that the basis of $\mathrm{gr}^{2} J$ obtained in [HS 2014] is too lengthy to handle. In fact, consider

$$
T_{1}:=\left\{t_{i}^{\prime} \mid 1 \leq i \leq n\right\} \cup\left\{t_{i j}^{\prime} \mid 1 \leq i<j \leq n\right\} \cup\left\{t_{i j k}^{\prime} \mid 1 \leq i<j<k \leq n\right\} \subset J
$$

and

$$
\begin{aligned}
T_{2}:= & \left\{t_{i}^{\prime} t_{j}^{\prime} \mid 1 \leq i \leq j \leq n\right\} \cup\left\{t_{i}^{\prime} t_{a b}^{\prime} \mid 1 \leq i \leq n, 1 \leq a<b \leq n\right\} \\
& \cup\left\{t_{i}^{\prime} t_{a b c}^{\prime} \mid 1 \leq i \leq n, 1 \leq a<b<c \leq n\right\} \\
& \cup\left\{t_{i j}^{\prime} t_{a b}^{\prime} \mid 1 \leq i<j \leq n, 1 \leq a<b \leq n,(i, j) \leq(a, b)\right\} \\
& \cup\left\{t_{a b}^{\prime} t_{a b c}^{\prime}, t_{a c}^{\prime} t_{a b c}^{\prime}, t_{b c}^{\prime} t_{a b c}^{\prime} \mid 1 \leq a<b<c \leq n\right\} \\
& \cup\left\{t_{i a}^{\prime} t_{a b c}^{\prime}, t_{i b}^{\prime} t_{a b c}^{\prime}, t_{i c}^{\prime} t_{a b c}^{\prime}, t_{i a}^{\prime} t_{i b c}^{\prime}, t_{a b}^{\prime} t_{i a c}^{\prime}, t_{a b}^{\prime} t_{i b c}^{\prime}, t_{a c}^{\prime} t_{i b c}^{\prime}, t_{i b}^{\prime} t_{i a c}^{\prime} \mid\right. \\
& \cup\left\{t_{j a}^{\prime} t_{i b c}^{\prime}, t_{j b}^{\prime} t_{i a c}^{\prime}, t_{j c}^{\prime} t_{i a b}^{\prime}, t_{a b}^{\prime} t_{i j c}^{\prime}, t_{a c}^{\prime} t_{i j b}^{\prime}, t_{b c}^{\prime} t_{i j a}^{\prime} \mid 1 \leq i<j<b<a<a<b\right\} \\
\subset & J^{2} .
\end{aligned}
$$


We showed in [HS 2014] that the $T_{k}$ form a basis of $\mathrm{gr}^{k} J$ for $k=1,2$. We cannot write down the total image of $\eta_{1}$ explicitly by hand, because $T_{2}$ consists of too many monomials.

Finally, we remark on the nontriviality of the coset class of $\eta$ in the first cohomology group of Aut $F_{n}$ with coefficients in $\operatorname{Hom}_{\mathbb{Q}}\left(\mathrm{gr}^{1} J, \mathrm{gr}^{2} J\right)$. Consider the automorphism $\sigma:=\left[K_{21}, K_{23}\right] \in \mathcal{A}_{n}(2) \subset \mathcal{E}_{n}(1)$. It satisfies

$$
x_{i}^{\sigma}= \begin{cases}{\left[x_{1}^{-1}, x_{3}^{-1}\right] x_{2}\left[x_{3}^{-1}, x_{1}^{-1}\right]} & \text { if } i=2, \\ x_{i} & \text { if } i \neq 2 .\end{cases}
$$

Hence we see that $\sigma$ maps $t_{123}^{\prime} \in \mathbb{Q}[t] / I$ to

$$
\begin{aligned}
t_{123}^{\prime}-2\left(t_{1}^{\prime}\right)^{2}-4 t_{1}^{\prime} t_{2}^{\prime}+4 t_{2}^{\prime} t_{3}^{\prime}+2\left(t_{3}^{\prime}\right)^{2}+2 t_{12}^{\prime} t_{1}^{\prime}-2 t_{12}^{\prime} t_{3}^{\prime}+6 t_{13}^{\prime} t_{1}^{\prime}+6 t_{13}^{\prime} t_{2}^{\prime} \\
+2 t_{13}^{\prime} t_{3}^{\prime}+2 t_{23}^{\prime} t_{1}^{\prime}-2 t_{23}^{\prime} t_{3}^{\prime}-4\left(t_{13}^{\prime}\right)^{2}-6 t_{12}^{\prime} t_{13}^{\prime}-2 t_{13}^{\prime} t_{23}^{\prime}+4 t_{13}^{\prime} t_{123}^{\prime}
\end{aligned}
$$

modulo $J^{3}$ by a hand calculation. By using the basis $T_{2}$ of $\mathrm{gr}^{2} J$, we can see that

$$
\tilde{\eta}_{1}(\sigma)\left(t_{123}^{\prime}\right)=\left(t_{123}^{\prime}\right)^{\sigma}-t_{123}^{\prime} \neq 0 \in \mathrm{gr}^{2} J \text {. }
$$

Thus the restriction $\tilde{\eta}_{1}$ of $\eta$ to $\mathcal{E}_{n}(1)$ is a nontrivial homomorphism, and so is the cohomology class of $\eta$ in

$$
H^{1}\left(\text { Aut } F_{n}, \operatorname{Hom}_{\mathbb{Q}}\left(\operatorname{gr}^{1} J, \mathrm{gr}^{2} J\right)\right) .
$$

It seems to be a natural and interesting problem to determine the above first cohomology group, and show whether $\eta$ generates it or not.

\section{The image of the crossed homomorphism $\eta$}

In order to calculate the image of $\eta$, it is important to know $\eta(\sigma)$ with $\sigma$ generators of $\sigma \in$ Aut $F_{n}$. In this section, we calculate the images of Nielsen's generators of Aut $F_{n}$ by the crossed homomorphism $\eta$.

For any $k \geq 2$, let $\rho_{k}$ : Aut $F_{n} \rightarrow \overline{\operatorname{Aut}}\left(J / J^{k}\right)$ be the natural homomorphism induced from the action of Aut $F_{n}$ on $J / J^{k}$. For any $\sigma \in$ Aut $F_{n}$, by identifying $\overline{\operatorname{Aut}}\left(J / J^{3}\right)$ with the semidirect product $\operatorname{Hom}_{\mathbb{Q}}\left(\operatorname{gr}^{1} J, \operatorname{gr}^{2} J\right) \rtimes \overline{\operatorname{Aut}}\left(J / J^{2}\right)$, we have

$$
\rho_{3}(\sigma)=\left(\eta(\sigma), \rho_{2}(\sigma)\right)=(\eta(\sigma), 1)\left(0, \rho_{2}(\sigma)\right)=(\eta(\sigma), 1) s\left(\rho_{2}(\sigma)\right) \in \overline{\operatorname{Aut}}\left(J / J^{3}\right),
$$

and hence

$$
(\eta(\sigma), 1)=\rho_{3}(\sigma) s\left(\rho_{2}(\sigma)\right)^{-1}
$$

From this, in order to compute $\eta(\sigma)$ for any $\sigma \in$ Aut $F_{n}$, it suffices to compute $\rho_{3}(\sigma) s\left(\rho_{2}(\sigma)\right)^{-1}$. Let $\gamma_{1}, \gamma_{2}, \ldots, \gamma_{p} \in J$ and $\gamma_{p+1}, \ldots, \gamma_{p+q} \in J^{2}$ be elements 
such that $\left(\left[\gamma_{1}\right]_{2},\left[\gamma_{2}\right]_{2}, \ldots,\left[\gamma_{p}\right]_{2}\right)$ and $\left(\left[\gamma_{p+1}\right]_{3}, \ldots,\left[\gamma_{p+q}\right]_{3}\right)$ form bases of $\operatorname{gr}^{1} \boldsymbol{J}$ and $\mathrm{gr}^{2} J$. By recalling the arguments in the previous section, we see that for any $1 \leq j \leq p$, if $\rho_{3}(\sigma)$ satisfies

$$
\rho_{3}(\sigma)\left(\left[\gamma_{j}\right]_{3}\right)=a_{1 j}\left[\gamma_{1}\right]_{3}+\cdots+a_{p j}\left[\gamma_{p}\right]_{3}+a_{p+1, j}\left[\gamma_{p+1}\right]_{3}+\cdots+a_{p+q, j}\left[\gamma_{p+q}\right]_{3}
$$

for some $a_{i j} \in \mathbb{Q}$, then

$$
s\left(\rho_{2}(\sigma)\right)\left(\left[\gamma_{j}\right]_{3}\right)=a_{1 j}\left[\gamma_{1}\right]_{3}+\cdots+a_{p j}\left[\gamma_{p}\right]_{3}
$$

for any $1 \leq j \leq n$. By using these facts, we can compute the images $\eta(\sigma)$ for any $\sigma \in$ Aut $F_{n}$.

Here let us recall Nielsen's generators of Aut $F_{n}$. Let $P, Q, S$ and $U$ be the automorphisms of $F_{n}$ defined as follows:

\begin{tabular}{ccccccc}
\hline & $x_{1}$ & $x_{2}$ & $x_{3}$ & $\cdots$ & $x_{n-1}$ & $x_{n}$ \\
\hline$P$ & $x_{2}$ & $x_{1}$ & $x_{3}$ & $\cdots$ & $x_{n-1}$ & $x_{n}$ \\
$Q$ & $x_{2}$ & $x_{3}$ & $x_{4}$ & $\cdots$ & $x_{n}$ & $x_{1}$ \\
$S$ & $x_{1}^{-1}$ & $x_{2}$ & $x_{3}$ & $\cdots$ & $x_{n-1}$ & $x_{n}$ \\
$U$ & $x_{1} x_{2}$ & $x_{2}$ & $x_{3}$ & $\cdots$ & $x_{n-1}$ & $x_{n}$ \\
\hline
\end{tabular}

Namely, $P$ is the automorphism of $F_{n}$ induced from the permutation of $x_{1}$ and $x_{2}$, $Q$ is induced from the cyclic permutation of the basis, and so on. Nielsen [1924] showed that Aut $F_{n}$ is generated by $P, Q, S$ and $U$ for any $n \geq 2$, and also gave finitely many relations among the generators $P, Q, S$ and $U$. This is the first finite presentation for Aut $F_{n}$.

By direct computation, we can see that $\rho_{3}(P) s\left(\rho_{2}(P)\right)^{-1}$ satisfies

$$
\begin{aligned}
t_{i}^{\prime} & \mapsto t_{i}^{\prime} \quad \text { for any } 1 \leq i \leq n, \\
t_{i j}^{\prime} & \mapsto t_{i j}^{\prime} \quad \text { for any } 1 \leq i<j \leq n, \\
t_{i j k}^{\prime} & \mapsto \begin{cases}t_{12 k}^{\prime}-\left\{t_{1}^{\prime} t_{2 k}^{\prime}+t_{2}^{\prime} t_{1 k}^{\prime}+t_{k}^{\prime} t_{12}^{\prime}-2\left(t_{1}^{\prime} t_{2}^{\prime}+t_{1} t_{k}^{\prime}+t_{2} t_{k}^{\prime}\right)\right\} & \text { if }(i, j)=(1,2), \\
t_{i j k}^{\prime} & \text { otherwise, }\end{cases}
\end{aligned}
$$

and hence obtain

$$
\eta(P)=-\sum_{k=3}^{n}\left(t_{12 k}^{\prime}\right)^{*} \otimes\left\{t_{1}^{\prime} t_{2 k}^{\prime}+t_{2}^{\prime} t_{1 k}^{\prime}+t_{k}^{\prime} t_{12}^{\prime}-2\left(t_{1}^{\prime} t_{2}^{\prime}+t_{1} t_{k}^{\prime}+t_{2} t_{k}^{\prime}\right)\right\},
$$

where $\left(t_{\bullet}^{\prime}\right)^{*}$ means the dual basis in $\operatorname{Hom}_{\mathbb{Q}}\left(\operatorname{gr}^{1} J, \mathbb{Q}\right)$ of $t_{\bullet}^{\prime}$ in $\mathrm{gr}^{1} J$. Similarly, we can obtain the equalities 


$$
\begin{aligned}
& \eta(Q)=0, \\
& \eta(S)=-\sum_{j=2}^{n}\left(t_{i j}^{\prime}\right)^{*} \otimes t_{1}^{\prime} t_{j}^{\prime}-\sum_{2 \leq j<k \leq n}\left(t_{1 j k}^{\prime}\right)^{*} \otimes t_{1}^{\prime} t_{j k}^{\prime}, \\
& \begin{aligned}
\eta(U)=-\left(t_{12}^{\prime}\right)^{*} \otimes t_{1}^{\prime} t_{2}^{\prime}-\sum_{k=3}^{n}\left(t_{12 k}^{\prime}\right)^{*} \otimes t_{2}^{\prime} t_{1 k}^{\prime} \\
+\sum_{3 \leq j<k \leq n}\left(t_{1 j k}^{\prime}\right)^{*} \otimes\left\{-\left(t_{1}^{\prime} t_{k}^{\prime}+t_{2}^{\prime} t_{j}^{\prime}+t_{j}^{\prime} t_{k}^{\prime}+2 t_{1}^{\prime} t_{j}^{\prime}+2 t_{2}^{\prime} t_{k}^{\prime}\right)\right. \\
+\left(t_{1}^{\prime} t_{2 j}^{\prime}+t_{1}^{\prime} t_{j k}^{\prime}-t_{2}^{\prime} t_{1 j}^{\prime}+t_{2}^{\prime} t_{j k}^{\prime}+t_{j}^{\prime} t_{12}^{\prime}+t_{j}^{\prime} t_{1 k}^{\prime}+t_{k}^{\prime} t_{12}^{\prime}+t_{k}^{\prime} t_{2 j}^{\prime}\right) \\
\left.-\frac{1}{2}\left(t_{1}^{\prime} t_{2 j k}^{\prime}-t_{2}^{\prime} t_{1 j k}^{\prime}+t_{j}^{\prime} t_{12 k}^{\prime}+t_{k}^{\prime} t_{12 j}^{\prime}\right)-\frac{1}{2}\left(t_{12}^{\prime} t_{j k}^{\prime}-t_{1 j}^{\prime} t_{2 k}^{\prime}+t_{1 k}^{\prime} t_{2 j}^{\prime}\right)\right\} .
\end{aligned}
\end{aligned}
$$

Since $P, Q, S$ and $U$ generate Aut $F_{n}$, by using the Leibniz rule, we can calculate $\eta(\sigma)$ for any $\sigma \in$ Aut $F_{n}$.

\section{Acknowledgments}

The author would like to thank the referee for the careful reading and helpful comments for the original paper. The author is supported by Grant-in-Aid for Young Scientists (B) by JSPS.

\section{References}

[Andreadakis 1965] S. Andreadakis, "On the automorphisms of free groups and free nilpotent groups", Proc. London Math. Soc. (3) 15 (1965), 239-268. MR 32 \#5746 Zbl 0135.04502

[Cartan and Eilenberg 1999] H. Cartan and S. Eilenberg, Homological algebra, Princeton University Press, 1999. MR 2000h:18022 Zbl 0933.18001

[Day 2013] M. B. Day, "Extensions of Johnson's and Morita's homomorphisms that map to finitely generated abelian groups", J. Topol. Anal. 5:1 (2013), 57-85. MR 3043442 Zbl 1270.57006

[Fricke and Klein 1897] R. Fricke and F. Klein, Vorlesungen über die Theorie der automorphen Funktionen, 1: Die gruppentheoretischen Grundlagen, Teubner, Leipzig, 1897. MR 32 \#1348 JFM 28.0334.01

[Hain 1997] R. Hain, "Infinitesimal presentations of the Torelli groups", J. Amer. Math. Soc. 10:3 (1997), 597-651. MR 97k:14024 Zbl 0915.57001

[Hatakenaka and Satoh 2014] E. Hatakenaka and T. Satoh, "On the graded quotients of the ring of Fricke characters of a free group", 2014. arXiv 1206.1500

[Hatakenaka and Satoh 2015] E. Hatakenaka and T. Satoh, "On the rings of Fricke characters of free abelian groups", J. Commut. Alg. (online publication November 2015). To appear.

[Horowitz 1972] R. D. Horowitz, "Characters of free groups represented in the two-dimensional special linear group”, Comm. Pure Appl. Math. 25 (1972), 635-649. MR 47 \#3542 Zbl 1184.20009

[Johnson 1980] D. Johnson, "An abelian quotient of the mapping class group $\mathcal{I}_{g}$ ”, Math. Ann. 249:3 (1980), 225-242. MR 82a:57008 Zbl 0409.57009

[Johnson 1983] D. Johnson, "The structure of the Torelli group, I: A finite set of generators for I'", Ann. of Math. (2) 118:3 (1983), 423-442. MR 85a:57005 Zbl 0549.57006 
[Johnson 1985a] D. Johnson, "The structure of the Torelli group, II: A characterization of the group generated by twists on bounding curves", Topology 24:2 (1985), 113-126. MR 86i:57011 Zbl 0571.57009

[Johnson 1985b] D. Johnson, "The structure of the Torelli group, III: The abelianization of $\mathcal{I}$ ", Topology 24:2 (1985), 127-144. MR 87a:57016 Zbl 0571.57010

[Kawazumi 2006] N. Kawazumi, "Cohomological aspects of Magnus expansions", 2006. arXiv math. GT/0505497

[Maclachlan and Reid 2003] C. Maclachlan and A. W. Reid, The arithmetic of hyperbolic 3-manifolds, Graduate Texts in Mathematics 219, Springer, New York, 2003. MR 2004i:57021 Zbl 1025.57001

[Magnus 1980] W. Magnus, "Rings of Fricke characters and automorphism groups of free groups", Mathematische Zeitschrift 170:1 (1980), 91-103.

[Morita 1993a] S. Morita, "Abelian quotients of subgroups of the mapping class group of surfaces", Duke Math. J. 70:3 (1993), 699-726. MR 94d:57003 Zbl 0801.57011

[Morita 1993b] S. Morita, “The extension of Johnson's homomorphism from the Torelli group to the mapping class group”, Invent. Math. 111:1 (1993), 197-224. MR 93j:57001 Zbl 0787.57008

[Nielsen 1924] J. Nielsen, "Die Isomorphismengruppe der freien Gruppen”, Math. Ann. 91:3-4 (1924), 169-209. MR 1512188 JFM 50.0078.04

[Satoh 2009] T. Satoh, "The cokernel of the Johnson homomorphisms of the automorphism group of a free metabelian group", Trans. Amer. Math. Soc. 361:4 (2009), 2085-2107. MR 2009i:20073 Zbl 1171.20024

[Satoh 2013a] T. Satoh, "First cohomologies and the Johnson homomorphisms of the automorphism group of a free group", J. Pure Appl. Algebra 217:1 (2013), 137-152. MR 2965912 Zbl 1286.20040

[Satoh 2013b] T. Satoh, "A survey of the Johnson homomorphisms of the automorphism groups of free groups and related topics", preprint, 2013. Submitted to Handbook of Teichmüller theory, volume $V$. arXiv 1204.0876

[Vogt 1889] H. Vogt, "Sur les invariants fondamentaux des équations différentielles linéaires du second ordre”, Ann. Sci. École Norm. Sup. (3) 6 (1889), 3-71. MR 1508833 JFM 21.0314.01

[Whittemore 1973] A. Whittemore, "On special linear characters of free groups of rank $n \geq 4$ ", Proc. Amer. Math. Soc. 40 (1973), 383-388. MR 48 \#428 Zbl 0248.20031

Received July 21, 2014. Revised October 24, 2014.

TAKAO SATOH

DEPARTMENT OF MATHEMATICS

FACULTY OF SCIENCE DIVISION II

TOKYO UNIVERSITY OF SCIENCE

1-3 KagURAZAKA

SHINJUKU-KU

TOKYO 162-8601

JAPAN

takao@rs.tus.ac.jp 


\title{
PACIFIC JOURNAL OF MATHEMATICS
}

\author{
msp.org/pjm
}

Founded in 1951 by E. F. Beckenbach (1906-1982) and F. Wolf (1904-1989)

\section{EDITORS}

Don Blasius (Managing Editor)

Department of Mathematics

University of California

Los Angeles, CA 90095-1555

blasius@math.ucla.edu

\author{
Paul Balmer \\ Department of Mathematics \\ University of California \\ Los Angeles, CA 90095-1555 \\ balmer@math.ucla.edu \\ Robert Finn \\ Department of Mathematics \\ Stanford University \\ Stanford, CA 94305-2125 \\ finn@math.stanford.edu \\ Sorin Popa \\ Department of Mathematics \\ University of California \\ Los Angeles, CA 90095-1555 \\ popa@math.ucla.edu
}

\author{
Vyjayanthi Chari \\ Department of Mathematics \\ University of California \\ Riverside, CA 92521-0135 \\ chari@math.ucr.edu \\ Kefeng Liu \\ Department of Mathematics \\ University of California \\ Los Angeles, CA 90095-1555 \\ liu@math.ucla.edu \\ Jie Qing \\ Department of Mathematics \\ University of California \\ Santa Cruz, CA 95064 \\ qing@ cats.ucsc.edu
}

\section{PRODUCTION}

Silvio Levy, Scientific Editor, production@msp.org

\section{SUPPORTING INSTITUTIONS}

ACADEMIA SINICA, TAIPEI

CALIFORNIA INST. OF TECHNOLOGY

INST. DE MATEMÁTICA PURA E APLICADA

KEIO UNIVERSITY

MATH. SCIENCES RESEARCH INSTITUTE

NEW MEXICO STATE UNIV.

OREGON STATE UNIV.

\author{
STANFORD UNIVERSITY \\ UNIV. OF BRITISH COLUMBIA \\ UNIV. OF CALIFORNIA, BERKELEY \\ UNIV. OF CALIFORNIA, DAVIS \\ UNIV. OF CALIFORNIA, LOS ANGELES \\ UNIV. OF CALIFORNIA, RIVERSIDE \\ UNIV. OF CALIFORNIA, SAN DIEGO \\ UNIV. OF CALIF., SANTA BARBARA
}

\author{
Daryl Cooper \\ Department of Mathematics \\ University of California \\ Santa Barbara, CA 93106-3080 \\ cooper@math.ucsb.edu \\ Jiang-Hua Lu \\ Department of Mathematics \\ The University of Hong Kong \\ Pokfulam Rd., Hong Kong \\ jhlu@maths.hku.hk \\ Paul Yang \\ Department of Mathematics \\ Princeton University \\ Princeton NJ 08544-1000 \\ yang@math.princeton.edu
}

These supporting institutions contribute to the cost of publication of this Journal, but they are not owners or publishers and have no responsibility for its contents or policies.

See inside back cover or msp.org/pjm for submission instructions.

The subscription price for 2015 is US \$420/year for the electronic version, and \$570/year for print and electronic.

Subscriptions, requests for back issues and changes of subscribers address should be sent to Pacific Journal of Mathematics, P.O. Box 4163, Berkeley, CA 94704-0163, U.S.A. The Pacific Journal of Mathematics is indexed by Mathematical Reviews, Zentralblatt MATH, PASCAL CNRS Index, Referativnyi Zhurnal, Current Mathematical Publications and Web of Knowledge (Science Citation Index).

The Pacific Journal of Mathematics (ISSN 0030-8730) at the University of California, c/o Department of Mathematics, 798 Evans Hall \#3840, Berkeley, CA 94720-3840, is published twelve times a year. Periodical rate postage paid at Berkeley, CA 94704, and additional mailing offices. POSTMASTER: send address changes to Pacific Journal of Mathematics, P.O. Box 4163, Berkeley, CA 94704-0163.

PJM peer review and production are managed by EditFLOW ${ }^{\circledR}$ from Mathematical Sciences Publishers.

\section{PUBLISHED BY}

\section{mathematical sciences publishers \\ nonprofit scientific publishing}

http://msp.org/

(C) 2015 Mathematical Sciences Publishers 


\section{PACIFIC JOURNAL OF MATHEMATICS}

Volume $275 \quad$ No. $2 \quad$ June 2015

A combinatorial characterization of tight fusion frames

MARCIN BOWNIK, KURT LUOTO and EDWARD RICHMOND

Combinatorics of finite abelian groups and Weil representations

295

KUnAL DUTTA and AMritANSHU PRASAD

Compact anti-de Sitter 3-manifolds and folded hyperbolic structures on

325 surfaces

FRANÇOIS GUÉRITAUd, FANNY KASSEL and MAXIME WOLFF

Circular handle decompositions of free genus one knots

361

FABIOLA MANJARREZ-GUTIÉRREZ, VÍCTOR NÚÑEZ and

ENRIQUE RAMÍREZ-LOSADA

A pointwise a-priori estimate for the $\bar{\partial}$-Neumann problem on weakly 409 pseudoconvex domains

R. MichaEL RANGE

Explicit Hilbert-Kunz functions of $2 \times 2$ determinantal rings

MARCUS ROBINSON and IRENA SWANSON

The Johnson-Morita theory for the ring of Fricke characters of free groups

\section{TAKAO SATOH}

Global representations of the conformal group and eigenspaces of the Yamabe operator on $S^{1} \times S^{n}$

MARK R. SEPANSKI and Jose A. Franco

Rota-Baxter operators on the polynomial algebra, integration, and averaging operators

Shanghua Zheng, Li GuO and MARKus Rosenkranz

Correction to the article Quiver grassmannians, quiver varieties and the preprojective algebra

Alistair Savage and Peter Tingley 